\title{
Partizipative Forschung mit Wohnungslosen
}

\section{Partizipative Studie zu der Akzeptanz der Unterkünfte und den Lebenslagen wohnungsloser Menschen in Hannover}

In den beiden zurückliegenden Jahren entstand in Hannover eine Studie zur Wohnungslosigkeit, angelegt als konsequent partizipativer Prozess von Menschen, die von Wohnungslosigkeit betroffen sind und jungen Fachkräften bzw. Studierenden aus der Sozialen Arbeit. Dabei wurden sowohl quantitative als auch qualitative Daten erhoben; in einem ersten Zwischenbericht wurden die quantitativen Ergebnisse präsentiert (vgl. armutstinkt.de 2020). Die Entstehungsgeschichte der Studie, die Herausforderungen und Chancen, die mit der partizipativen Forschungsmethode einhergehen und die besonderen Wissensbestände, die dadurch generiert werden konnten, werden in diesem Beitrag beschrieben.

$\mathrm{P}$ artizipation ist aus dem Sprachgebrauch der Sozialen Arbeit nicht mehr wegzudenken. Sie wurde in den letzten Jahrzehnten fest in die Leitbilder und Konzepte sozialer Organisationen und auch in die Rechtsprechung (z. B. $\$ 8$ SGB VIII) implementiert. In der Wohnungslosenhilfe hat sich allerdings „bis heute ein weitgehend bürgerliches und patriarchales System erhalten“" (Szynka 2010, S. 41), das eine paternalistische und defizitorientiere Haltung seitens der Sozialarbeiter innen begünstigen kann. So ist es wenig verwunderlich, dass Gerull zu dem Schluss kommt, das Berliner Wohnungslosenhilfesystem weise in Bezug auf Partizipation einen „blinden Fleck“ auf (vgl. Gerull 2018a, S. 41).
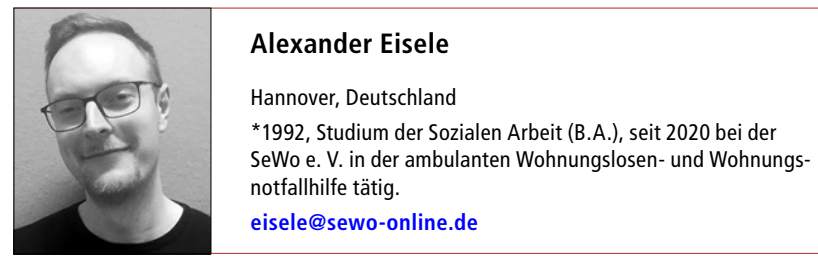

Zusammenfassung Kooperative Studien, also Studien, die konsequent die Betroffenen - hier: wohnungslose Menschen einbeziehen, erweitern das Wissen über den Nutzen und die Nutzbarkeit konkreter sozialer Einrichtungen und allgemeiner Lücken im sozialen System. Die an der Studie beteiligten Betroffenen klagten über konstante Bedrohung durch Gewalt, fehlenden Zugang zu medizinischer Regelversorgung (trotz Krankenversicherung), fehlende private Rückzugsräume (wie z. B. Einzelzimmer), die für den Rest der Bevölkerung „trivial als auch elementar“ sind, obdachlosen Menschen aber „strukturell verwehrt" werden.

Schlüsselwörter Obdachlosigkeit, Obdachlosenunterkünfte, Partizipative Forschung, Lebenslagen

\section{Partizipation als Randerscheinung} in der Wohnungslosenhilfe

Durch unsere Feldbeobachtungen, durch Praktika und Projekte während des Studiums, kamen wir ${ }^{1}$ über das Hilfesystem in Hannover zu einer vergleichbaren Einschätzung. Deshalb entschlossen wir uns im letzten Semester unseres Studiums, ein Konzept für ein partizipatives Modellprojekt zu entwickeln, das wir seit dem 1. April 2020 während unseres Anerkennungsjahrs durchführen. Wir verstehen Partizipation im Sinne von Kunstreich als grundlegendes Arbeitsprinzip, das nicht nur punktuell zum Einsatz kommt, sondern Kernbestandteil unserer Arbeit ist (vgl. Kunstreich 2014, S. 266). Durch eine fest verankerte Mitbestimmung der Nutzer_innen innerhalb des Unterstützungsprozesses sollen Selbstwirksamkeitserfahrungen ermöglicht werden, wodurch gesellschaftliche Teilhabe (wieder-) erlangt werden kann.

\section{Partizipative Forschung als Teil des Modellprojekts} Der partizipative Ansatz beschränkt sich allerdings nicht nur auf die individuellen Unterstützungsprozesse von Nutzer_innen, sondern ist auch fester Bestandteil von Gruppenarbeitsprozessen und öffentlichkeitswirksamen Aktionen im Sozialraum (vgl. zum Arbeitsprinzip Sozialraumorientierung Oelschlägel 2002, S. 9). Dies gilt auch für die erwähnte Studie. Die Arbeiten daran begannen im Herbst 2019 und wurden im Frühjahr 2020 in unser Modellprojekt überführt. Das Forschungsteam bestand zunächst aus Axel Fleischhauer, dessen Bachelorarbeit das partizipative Forschungsprojekt darstellte, und drei Personen, die akut von Wohnungslosigkeit betroffen sind: ein Mann und eine Frau, 
die sich gemeinsam eine „Platte“ teilten und Biwak machten sowie ein Mann, der ohne eigenen Mietvertrag bei einem Bekannten unterkommt. Fleischhauer und ein Kommilitone (der später auch Teil des Forschungsteams wurde) hatten im Rahmen eines studentischen Projekts gemeinsam mit den Betroffenen den „Runden Tisch Forum für Betroffene von Wohnungslosigkeit“" (mittlerweile umbenannt in ,armutstinkt.de“) aufgebaut. Dieser ist zum einen eine Austauschplattform für Betroffene und soll zum anderen als Grundlage dienen, aus diesem Zusammenhang heraus eine Stimme der Betroffenen zu entwickeln, die die Betroffenenperspektive in den stadtgesellschaftlichen Diskurs mit einbringt. Zu Beginn der Studie konnte das Forschungsteam also bereits auf eine eineinhalbjährige erfolgreiche Zusammenarbeit zurückblicken, die den Forschungsprozess sicherlich positiv beeinflusste. Seit Winter/Frühjahr 2020 besteht das Forschungsteam aus vier jungen Fachkräften aus der Wohnungslosenhilfe und fünf erfahrenen Forschungsmitgliedern ${ }^{2}$.

\section{Zielsetzung der Studie}

In Hannover gibt es ein großes Akzeptanzproblem der städtischen Obdachlosenunterkünfte. Obwohl ungefähr 100 freie Plätze in den Unterkünften zur Verfügung stehen, ziehen es mehrere Hundert Personen in Hannover vor, auf der Straße zu schlafen. Durch eine leicht verbesserte Ausstattung der Unterkünfte versuchte die Stadt Hannover, diese Problematik zu beheben (vgl. Hannover 2019, 2019a, 2019b). Dies war der Ausgangspunkt für die Studie. Das sich nun formierende Forschungsteam stellte die Vermutung auf, dass die eingeleiteten Maßnahmen der Stadt an den tatsächlichen Bedarfen der Betroffenen vorbeigingen und sich die Akzeptanzprobleme auf diese Art und Weise nicht beheben lassen würden. Aus dieser Vermutung kristallisierten sich folgende Forschungsfragen heraus:

1. Bestehen die Akzeptanzprobleme weiter, obwohl die Stadt seit dem vorangegangenen Winter nach eigener Aussage bereits begonnen hat, die Probleme in den Einrichtungen anzugehen?

2. Wo sehen Betroffene die größten Probleme in den Einrichtungen und den stärksten Handlungsbedarf?

Neben diesen beiden Forschungsfragen sollten auch die allgemeine Lebenssituation der Betroffenen beforscht werden.

\section{Methodisches Vorgehen}

Zentrale methodische Grundlage dieser Studie ist der konsequent partizipative Anspruch aller Beteiligten.
„Konsequent partizipativ“ meint, dass hier der gesamte Forschungsprozess von erfahrenen Forschungsmitgliedern und Fachkräften gemeinsam konzipiert, durchgeführt und interpretiert wurde und in einem diskursiven Prozess entsprechende Schlussfolgerungen gezogen sowie Forderungen erhoben wurden. Diese Forschungsmethode wurde zuletzt von der Forschungsgruppe um Hella von Unger für den deutschsprachigen Raum aufgearbeitet (vgl. Unger 2014).

Darüber hinaus wurde die Studie in ihren einzelnen Bestandteilen im Tandem-Prinzip mit je einem erfahrenen Forschungsmitglied sowie einem Sozialarbeitenden verfasst und die Ergebnisse anschließend in der Gruppe diskutiert und ggf. angepasst. Die Zielsetzung dieses Forschungsstils ist zweiteilig. Es sollen zum einen differenziertere Wissensbestände generiert werden, als die klassische Forschung es mit ihrer „Objektivierung der Subjekte“ leisten könnte. Dies wird mit dem durchgängig diskursiven Prozess zwischen den Fachkräften und den erfahrenen Forschungsmitgliedern erreicht. Durch das Einbringen der Perspektive und Expertise der erfahrenen Forschungsmitglieder während des gesamten Prozesses entsteht ein Praxiswissen, das auf herkömmliche Art nicht zu explorieren ist. Zum anderen soll die Forschung nicht nur einem Selbstzweck dienen, sondern direkt in die Lebenswelten wirken und die Betroffenen auf diese Weise empowern. Ähnlich wie beim Ansatz der Interventionsforschung (vgl. Lerchster 2012, S. 23-73), geht es hierbei um Selbstermächtigung durch Selbstreflexion, jedoch erweitert um die Aktion und einen Kompetenzerwerb der Betroffenen.

\section{Erhebung der Daten}

Für die Studie fand sowohl eine qualitative als auch eine quantitative Datenerhebung statt. Für die quantitative Erhebung diente die Lebenslagenstudie von Gerull (vgl. Gerull 2018b) als Vorbild. Zum einen ist dadurch ein Vergleich der von uns erhobenen Daten möglich und zum anderen überzeugte uns der Lebenslagenindex der Studie. Durch diesen werden nicht nur die vermeintlich objektivierbaren Lebensumstände der Befragten erfasst, sondern auch die subjektiven Empfindungen der Befragten zu einzelnen Lebensbereichen (vgl. Gerull 2018b, S. 5 und 6). Der von der Forschungsgruppe um Gerull entwickelte Fragebogen wurde, mit kleinen Modifikationen, übernommen. Da aber wie bereits beschrieben die Akzeptanzprobleme der Unterkünfte mit dieser Studie erforscht werden sollten, wurde für die Unterkünfte ein zweiter Teil des Fragebogens neu konzipiert. Die Erhebung erfolgte vom 14.12.2019 bis zum 14.02.2020. Insgesamt wurden 118 Fragebögen ausgefüllt, von denen 105 für diese Studie ausgewertet werden konnten. 


\section{Durchblick: Partizipation in der Wohnungslosenhilfe}

Die qualitative Erhebung wurde am 14.12.2019 durchgeführt. Es fand eine Veranstaltung statt, an der rund 40 Personen, die von Wohnungslosigkeit betroffen sind, aktiv teilgenommen haben. Dabei wurde die Methode „World Café" angewandt. Da die Ergebnisse dieser Veranstaltung noch nicht veröffentlicht wurden, wird an dieser Stelle nicht weiter darauf eingegangen.

\section{Zwischenbericht}

Die COVID-19-Pandemie ging auch an unserer Forschungsgruppe nicht spurlos vorbei. Aus verschiedenen Gründen fanden zwischen März und Mai 2020 keine Forschungstreffen statt. Des Weiteren hatte die Pandemie erstaunlicherweise auch positive Effekte auf das Wohnungslosenhilfesystem in Hannover. Das ansonsten sehr starre System bekam neue Impulse und neue Unterbringungsformen konnten genutzt werden. So wurden für vier Monate ungefähr 100 Betroffene in der Jugendherberge Hannover in Einzelzimmern untergebracht (vgl. HAZ 2020). Weitere 30 Betroffene konnten in Hotels in Einzelzimmern untergebracht werden. Um Einfluss auf den politischen Diskurs nehmen zu können und in dieser Situation des vermeintlichen Umbruchs die große Unzufriedenheit mit den regulären Unterkünften der Stadt aufzeigen zu können, entschlossen wir uns, die Ergebnisse unserer quantitativen Erhebung möglichst schnell in Form eines Zwischenberichtes zu veröffentlichen.

\section{Ergebnisse des Zwischenberichts}

Das zentrale Ergebnis ist die große Unzufriedenheit der befragten Personen, die sich sowohl im Abschnitt der Lebenslagen (z. B. mit der derzeitigen Wohn- und Erwerbssituation) als auch im Abschnitt zu den Unterkünften (z. B. mit den hygienischen Zuständen innerhalb der Unterkünfte oder den Umgangsformen des Sicherheitspersonals) zeigt. Bei der Betrachtung im Einzelnen erscheint diese Unzufriedenheit als absolut nachvollziehbar. So ergab die Studie unter anderem, dass die Befragten einer massiven Bedrohungslage ausgesetzt sind und Gewalt eine prägende Konstante des Alltages vieler ist. Vielen Betroffenen bleibt der Zugang zur medizinischen Regelversorgung trotz Krankenversicherung aus verschiedenen Gründen verwehrt. Eine Unterbringung in einem Einzelzimmer steht nur einer kleinen Minderheit zur Verfügung. Private Rückzugsorte, die für die sogenannte Mehrheitsgesellschaft sowohl trivial als auch elementar sind, werden den Betroffenen somit strukturell verwehrt. Auch die Ergebnisse zu der geleisteten Sozialen Arbeit in den Unterkünften offenbaren erheblichen Nachbesserungsbedarf, was sowohl die Erreichbarkeit der Sozialarbeiter_innen als auch deren fachliche Kompetenz betrifft.

\section{Forderungen der Studie}

Die zentrale Forderung der Studie, die sich für die Forschungsgruppe aus den Ergebnissen ableitet, ist, dass es ein Grundrecht auf Wohnen geben muss. Dies impliziert, dass das präventive Netz gestärkt werden muss, um neue Wohnungslosigkeit zu verhindern. Des Weiteren ist es unbedingt notwendig die Standards innerhalb der Unterkünfte anzuheben und die Unterkünfte fachlich so auszurichten, dass sie lediglich als Übergangslösung fungieren. Eine Anhebung der Standards meint konkret, dass jeder Person eine Einzelunterbringung mit eigener Nasszelle zur Verfügung gestellt werden muss. Alle Betroffenen sollten zudem in für sie angemessene Wohnformen vermittelt werden. Dafür muss dringend entsprechender Wohnraum zur Verfügung gestellt werden. Mittelfristig müssen Wohnungs- und Obdachlosigkeit also überwunden werden, anstatt diese weiter $\mathrm{zu}$ verwalten.

\section{Herausforderungen der Methodik}

Der partizipative Forschungsansatz birgt neben seinen Chancen, auf die weiter unten eingegangen wird, auch einige Herausforderungen, die bei der Planung entsprechender Forschungsprojekte berücksichtigt werden müssen. Es muss mit einem erhöhten Zeitaufwand gerechnet werden. Dies betrifft zum einen die Planung. Hierbei gilt es nicht nur zu schauen, welche Forschungsmethoden wissenschaftstheoretisch und -praktisch geeignet sind, die Forschungshypothesen zu überprüfen, sondern man muss auch abschätzen, welche Methoden für die erfahrenen Forschungsmitglieder angemessen und umsetzbar sind. Hierfür müssen verschiedene Möglichkeiten vorgestellt werden und wissenschaftstheoretische Kompetenzen müssen vermittelt werden, um eine partizipative Auswahl des Studiendesigns zu ermöglichen. Generell durchzieht die Kompetenzvermittlung den gesamten Forschungsprozess. Der Wissenstransfer fließt bei partizipativen Projekten aber niemals nur in eine Richtung, denn das Prinzip der Perspektivverschränkung führt bei allen Beteiligten zwangsläufig zu einer Perspektiverweiterung.

Durch das von uns gewählte Tandem-Prinzip und die anschließende Diskussion des Ergebnisses in der Forschungsgruppe muss mit erhöhtem Zeitaufwand gerechnet werden. Außerdem bedarf es innerhalb der Forschungsgruppe einer Übereinkunft bezüglich der Gleichwertigkeit der unterschiedlichen Wissensbestände. Alle Beteiligten müssen akzeptieren, dass keine Hierarchisierung zwischen der Sozialarbeitswissenschaft und dem Praxiswissen der erfahrenen Forschungsmitglieder vorgenommen werden darf. Ferner muss ehrlich und transparent mit den gesellschaftlichen Positionen 
der einzelnen Mitglieder und den damit einhergehenden Machtverhältnissen umgegangen werden. Für die Einhaltung dieser Prinzipien bedarf es, insbesondere für die Sozialarbeitenden, einer fortlaufenden (Selbst-) Reflexion und einer offenen Kommunikation in der Gruppe. Ansonsten droht die Forschung in ein scheinpartizipatives Projekt abzudriften.

Neben den bereits erwähnten Herausforderungen müssen bei der Zusammenarbeit mit von Wohnungslosigkeit Betroffen zusätzlichen Hürden einkalkuliert werden. Wohnungslosigkeit und insbesondere Obdachlosigkeit ist eine existenzielle Ausnahmesituation. Vermeintlich triviale Dinge wie Regen können dazu führen, dass ein Forschungstreffen nicht stattfinden kann, da die Platte nicht abgebaut werden kann und die erfahrenen Forschungsmitglieder somit bewegungsunfähig sind. Auch kann die geplante Tagesordnung eines Treffens spontan geändert werden müssen, da tagesaktuelle Geschehnisse in den Mittelpunkt rücken. Es bedarf also einer ausreichenden Flexibilität inhaltlicher, aber auch organisatorischer Art.

$\mathrm{Zu}$ betonen ist, dass Engagement und Ehrgeiz der erfahrenen Forschungsmitglieder bemerkenswert waren. Obwohl wir für das Projekt keine Forschungsgelder akquirieren konnten und somit auch keine Vergütung der Erfahrenen stattfinden konnte, fand so gut wie jedes Forschungstreffen statt. Dies ist umso erstaunlicher, wenn berücksichtigt wird, dass zwei Mitglieder in einem Zelt überwintern mussten.

\section{Chancen der Methodik}

Die erfahrenen Forschungsmitglieder können durch den Ansatz individuelle Entwicklungsprozesse in verschieden Bereichen anstoßen bzw. ausbauen, so etwa in den Bereichen Kommunikation innerhalb der Gruppe und Außendarstellung, wissenschaftlicher und persönlicher Kompetenzen. Dieser Prozess kann zu einem differenzierteren Selbstbewusstsein führen. Des Weiteren kann durch eine wissenschaftliche Publikation, an der entscheidend mitgewirkt wurde, Selbstvertrauen gestärkt werden, insbesondere wenn die Publikation medial wahrgenommen wird (vgl. NP 2020a). Da die Initiative armutstinkt.de im Anschluss an die Veröffentlichung als ernstzunehmender Faktor im städtischen Diskurs wahrgenommen wurde, der eigene Demonstrationen organisiert (vgl. NP 2020b) und Kommunalpolitiker_innen beim Erstellen von Anträgen berät, kann von einer Selbstwirksamkeitserfahrung und einem geglückten Empowerment-Prozess gesprochen werden. Außerdem können die neu erworbenen Kompetenzen auf andere Projekte übertragen werden. So führte armutstinkt.de im Dezember 2020 im Auftrag des Fach- bereichs Soziales der Stadt Hannover eine kleine Umfrage unter Betroffenen von Wohnungslosigkeit durch, die zeigen sollte, ob die Anschaffung eines Duschbusses aus Betroffenenperspektive als sinnvoll erachtet wird.

Trotz des erhöhten Zeitaufwandes und der benötigten strukturierten Offenheit (vgl. Grundwald und Thiersch 2016, S. 11) eröffnet der konsequent partizipative Forschungsansatz viele Chancen für alle Beteiligten. Durch den andauernden diskursiven Prozess wurde es uns ermöglicht, sehr tief in die Lebensrealität der Erfahrenen einzutauchen. Durch diese Perspektiverweiterung konnten z. B. die strukturellen Gewalterfahrungen, denen die Betroffenen von Wohnungslosigkeit tagtäglich ausgesetzt sind und an denen auch die Soziale Arbeit oft nicht unbeteiligt ist, besser nachvollzogen werden. Die Perspektiverweiterung wirkt sich ebenfalls positiv auf die Einzelfallarbeit mit anderen Betroffenen aus. Auch führt die Akzeptanz der Gleichwertigkeit der Wissensbestände dazu, paternalistischen Entgleisungen im Arbeitsalltag entgegenzuwirken.

Auch die Studie profitierte qualitativ von der gewählten Methodik. Durch die Perspektivverschränkungen konnten Wissensbestände generiert werden, die durch ein rein sozialarbeitswissenschaftliches Forschungsteam nicht zu Tage gefördert worden wären. So sind z. B. die vorgenommenen Ergänzungen des Lebenslagenfragebogens von Gerull größtenteils auf die Anmerkungen der erfahrenen Forschungsmitglieder zurückzuführen. Dies führte dazu, dass der Fragebogen stärker an der Lebensrealität der Betroffenen ausgerichtet wurde. Zudem führte die gemeinsame Interpretation der Forschungsergebnisse zu einer differenzierteren Analyse, die durch eine ständige Vergegenwärtigung der Betroffenenperspektive aufgewertet wird. Ein nicht zu vernachlässigender Aspekt ist der besondere Feldzugang durch die erfahrenen Forschungsmitglieder, wodurch auch Teilnehmer_innen erreicht werden konnten, die keinerlei oder kaum Berührungspunkte mit dem Hilfesystem haben.

\section{Abschließende Bemerkungen}

Wie der Beitrag zeigt, ist der konsequent partizipative Forschungsansatz, trotz seines unter Umständen herausfordernden Zeitaufwandes, äußerst gewinnbringend für alle Beteiligten. Wenn die Profession Soziale Arbeit ihre eigenen Leitsätze ernstnimmt und Nutzer_innen wirklich als Expert_innen ihrer eigenen Lebenswelt anerkennt, muss eine wesentliche stärkere Beteiligung an der sozialarbeiterischen Praxis und am Wissenschaftsbetrieb erfolgen. Es ist an der Zeit, mehr Partizipation zu wagen. 
Eingegangen. 22. Dezember 2020

Angenommen. 15. Januar 2021

1. Am Projekt waren Axel Fleischhauer und Florian Schulz beteiligt, die auch an diesem Beitrag mitgewirkt haben.

2. Im Folgenden wird der Terminus ,erfahrene Forschungsmitglieder" bzw. „Erfahrene“ verwendet, um den speziellen Kompetenzen dieser Forschungsmitglieder gerecht zu werden und eine Abgrenzung zu den im allgemeinen als „Betroffene“ bezeichneten Menschen deutlich zu machen.

\section{Literatur}

armutstinkt.de (2020). „Das ist nicht mehr das eigene Leben, welches man lebt. " Partizipative Studie zu der Akzeptanz der Unterkünfte und den Lebenslagen wohnungsloser Menschen in Hannover (Erster Zwischenbericht). Online verfügbar unter Studie zu Lebenslagen und Zufriedenheit mit den Unterkuenften_Erster Zwischenbericht (armutstinkt.de).

Gerull, S. (2018a). „Spaghetti oder Reis?“. Partizipation in der Wohnungslosenhilfe. Berlin, Milow, Strasburg: Schibri.

Gerull, S. (2018b). 1. systematische Lebenslagenuntersuchung wohnungsloser Menschen. https://opus4.kobv.de/opus4-ash/frontdoor/deliver/index/ docId/246/file/ASH+EBET_Lebenslagenuntersuchung_2018.pdf. Zugegriffen: 20. Dez. 2020.

Grundwald, K., \& Thiersch, H. (2016). Vorwort. In K. Grunwald \& H. Thiersch (Hrsg.), Praxishandbuch lebensweltorientierte Soziale Arbeit (Bd. 3, S. 11-13). Weinheim, Basel: Beltz Juventa.

Hannover (2019). Sitzung Stadtbezirksrat Mitte am 16.09.2019. https://egovernment.hannover-stadt.de/lhhsimwebre.nsf/TM/20190916_STBR01. Zugegriffen: 20. Dez. 2020.

Hannoversche Allgemeine [HAZ] (2019a). Hannover startet Winternotprogramm für Obdachlose. https://www.haz.de/Hannover/Aus-der-Stadt/ Hannover-startet-Winternotprogramm-fuer-Obdachlose. Zugegriffen: 20 Dez. 2020.

Hannoversche Allgemeine [HAZ] (2019b). Obdachloser in Hannover: „Der Winter wird schrecklich“. https://www.haz.de/Hannover/Aus-derStadt/Obdachlose-in-Hannover-Elend-auf-Hannovers-Strassen-nimmt-zu. Zugegriffen: 20. Dez. 2020.

Hannoversche Allgemeine [HAZ] (2020). Obdachlose müssen Jugendherberge verlassen - doch wo sollen sie hin? https://www.haz.de/Hannover/ Aus-der-Stadt/Jugendherberge-Hannover-Stadt-findet-keine-neue-Unterkunft-fuer-Obdachlose. Zugegriffen: 20. Dez. 2020.

Kunstreich, T. (2014). Blicke auf die Jahre 1955, 1970 und 1995 sowie ein Rückblick auf die Soziale Arbeit in der DDR (5. Aufl.). Bielefeld: Kleine. 2 Bände, Grundkurs Soziale Arbeit. Sieben Blicke auf Geschichte und Gegenwart Sozialer Arbeit, 2

Lerchster, R. E. (2012). Zentrale Grundannahmen der Interventionsforschung. In L. Krainer \& R. E. Lerchster (Hrsg.), Paradigmen, Methoden, Reflexionen. Interventionsforschung, (Bd. 1, S. 23-73). Wiesbaden: Springer VS.

Neue Presse [NP] (2020a). Studie veröffentlicht: So viele Obdachlose leben in Hannover. https://www.neuepresse.de/Hannover/Meine-Stadt/armutstinkt.de-Studie-zur-Wohnungslosigkeit-in-Hannover. Zugegriffen: 20. Dez. 2020.

Neue Presse [NP] (2020b). Protest von „armutstinkt“ für bezahlbare Wohnungen. Printausgabe der Neuen Presse vom 11. Sept. 2020. Online nicht verfügbar.

Oelschlägel, D. (2002). Grundlagen der Gemeinwesenarbeit. Paradigmenwechsel. http://fc.quarternet.de/download/FOV1-0000D4EA/71-oelschlaegel.pdf. Zugegriffen: 21. Dez. 2020.

Szynka, P. (2010). Partizipation und (Selbst)-organisation in der Wohnungslosenhilfe. Wohnungslos, 2, 41-44.

von Unger, H. (2014). Partizipative Forschung. Wiesbaden: Springer. 
Hier steht eine Anzeige.

\section{Springer}

